\title{
A Practical Framework and approach for Privacy Issues Concerning Social Networking Sites
}

\author{
Mukesh Kumar \\ Department of Computer \\ Science and Engineering \\ MITM \\ Selaqui, Dehradun \\ Uttarakhand, India
}

\author{
Nupur Sharma \\ Department of Computer \\ Science and Engineering \\ MITM \\ Selaqui, Dehradun \\ Uttarakhand, India
}

\author{
Mitul Yadav \\ Department of Computer \\ Science and Engineering \\ Graphic Era Hill University \\ Bhimtal \\ Uttarakhand, India
}

\begin{abstract}
Social networking sites facilitate and develops a virtual community for people to share their thoughts, interest activities or to increase their horizon of camaraderie. With the advancement in Social networking sites or we can refer as online social networks the user can connect to each other. They can share pictures, videos and view to each other. With all these options available on SNS it creates a virtual world for the users. Because of all these reasons growth of SNS in recent years indicates that they are now mainstream communication technology for many people. Social networking sites come under few of the most frequently browsed categories websites in the world. However SNS are also vulnerable to various problems, threats and attacks such as disclosure of information, identity thefts etc. Privacy practices in social networking sites often appear convoluted as information sharing stands in discord with the need to reduce disclosure-related abuses. Facebook is one such most popular and widely used SNS which has its own strong set of Privacy policies. Yet they are also prone to various privacy issues and attacks. In this work we examine the currently existing privacy policies of Facebook since 2013 and then provide with certain new policies that enhance and strengthen the existing ones. We conclude with a discussion of how these findings may be integrated into the design of Facebook like SNS systems to facilitate interaction while enhancing individual privacy.
\end{abstract}

\section{Keywords}

Social networking sites (SNS's), Cyber stalking, Cyber bullying

\section{INTRODUCTION}

A social networking service is an online service, platform, or site that focuses on fascinating the building of social relations among people who, for example, activities, share interests, background, or real-life connections[7]. Advancement on Online Social Networks the user can connect with their friends very easily. They can share data, or view Pictures, Videos of each other. Because of all these reasons growth of SNS in recent years indicates that they are now a mainstream communications technology for many people[8]. The people who are using social networking sites see them as fun and easy leisure activities. By enhancing their social circle, users have the opportunity to commune with people who have the same interests. In today's scenario SNS are most widely used websites in world, with Facebook being the second most visited site on internet world wide first being Google. As on July 2013, Facebook has more than 1.20 billion active users[1] and the revenue generated by Facebook alone is 3.91(approx) million dollars[1]. Facebook is good for engaging consumers and consequently gaining valuable momentum of oral communication. The main way to get involved is to make a fan page that offers something valuable to the consumer like interesting group conversations, tools or casual games for convenience. The end-goal for gaining oral communication value is to get consumers to "like" your fan page. When they do, it's announced on their feed to all their friends and the fan gets regular updates from the brand. While brand can do much more on the Facebook platform than on Twitter and Youtube, in the end most successful initiatives have been driven by promotions, similar to Twitter. Here privacy is an important possession. We find it difficult to stifle our curiosity about others [10].Today SNS's is just not an entertainment websites, but one of the most important communication medium in today's world. Nevertheless there have been always a privacy and secrecy concerns about misusing the crucial information by internet perpetrators. Also SNS's are a perfect platform for virus authors to spread their malwares faster than traditional methods.

Facebook scams recently hits the headlines in the Internet fraud world. The Wall Street Journal [10] released an alarming reported, sending several into a frenzy regarding the privacy of their Facebook profiles. What WSJ found is that several Facebook apps are sending user Ids to third parties, like advertising networks. Facebook issued a statement for these privacy concerns saying" This is a more complicated technical challenge addressed last spring on facebook.com. So from all these secrecy issues and discussion privacy is in apex. By providing a set of privacy policies we can control the access of the information content that is available. While studying and exploring various privacy issues related to SNS's, we found that the current preventive measures suggested or applied on Facebook are still vulnerable and probably not sufficient for the user to control the access of their data. In this research paper we have surveyed the existing privacy policies of Facebook and found some vulnerability on these policies .Additionally we have proposed some new privacy policies to strengthen the existing ones. As a conclusion we have integrated our policies into a Facebook like SNS's system for enhancing individual privacy.

\section{BACKGROUND}

Social networking service [7] is an platform, online service or site that focuses on facilitating the building of social networks and relations among people who can share their interests, activities, real life connections. A social network services consists of a representation of each users profile, his/her social links, and a variety of additional services. Social network service usually means an individual-centered service whereas online community services are group centered. Geocities was the first web-based social networking site develop in 1994. In 1995 THEGLOBE.COM emerged allowing users to publish their content and interact with the 
others users sharing same interests. In 1997 AOL INSTANT MESSANGER was launched which popularized the concept of instant messaging. SIXDEGREES.COM was also launched in the same year which allowed users to create their individual profiles and listing friends.

Friendstar was the real breakthrough in the field of social networking. Launched in 2002, Friendstar was the pioneer of using the concept of online networking between the real friends. In 2003 MYSPACE was launched which was a Friendstar clone at the initial phase but still popular in those years. In 2004, Harvard University as a way of connecting all the U.S. college students launched FACEBOOK.

Many type of Social networks available. This fact sheet examines privacy and security implications of using a few of them. While this fact sheet does not address every type of social networks, many of the security and privacy recommendations are applicable to other type of networks.

- Personal Networks- This networks permit users to create detailed online profiles and connect with other users. These involve users to share one's gender, age, employment, personal contact information as well as file and links to music, photos, videos where sharing person is authorized or not.

- Status Update Networks- These are designed to permit users to post short status updates in order to communicate with other users rapidly. For example, Google Buzz Twitter. There may be privacy settings to restrict access to status updates.

- Location Networks- With the advent of GPSenabled cellular phones, location based networks are gaining popularity. These networks are designed to broadcast one's real-time location, either public information or as an update viewable to authorized contacts. Some examples include Brightkite, Foursquare, Loopt and Google Latitude.

- Content -Sharing Networks- This is a sharing based social networks which is designed to share music, photograph, videos. When these websites introduce the ability to create personal profiles, establish contacts and interact with other users, they become social networks. Some popular content sharing networks are Flickr, Youtube, thesixtyone.

- Shared-Interest Networks- Some social networks are built for a common interest for specific group of people. These are slanted toward a common subset of individual, such as those having common hobbies, educational backgrounds, religious views, political affiliations, sexual orientations or other defining interests. Examples include LinkedIn, Goodreads Black Planet and Gay.com.

\section{PRIVACY ISSUES IN SOCIAL NETWORKING SITES}

Social networking sites can control accessing users to their own profile but they cannot control what others may represent about them. Friends can sometimes disclose more information about the users than they should hence we can say them untrustworthy. Concept of third party application in today's SNS's also pose severe privacy threat. Without allowing the permission to access all of their public and private data, SNS's users cannot add an application to their profile. Facebook additionally gives third parties second degree access, for example when Robert installs a third party application it can also request information about his friends and network members. It is shown that only $9.3 \%$ of Facebook application require access to private data[3]. Advertising is the main source of income in most of the SNS's including Facebook. These SNS's allow merchants and third parties to take advantage of user information without their agreement. Some important privacy issues in Social networking sites are[1]

- SNS's currently available today does not aware use of the risks of revealing their personal information

- The privacy tool are very complex to understand and to use them due to which people feed up and they end up doing nothing.

- Users can control who can access what is in their profile but cannot control what others reveal about you.

- Privacy tools or options can provide options with "Friends" ," Friends of Friends" or "Everyone", with Facebook providing much more facility to customize number of options according to which friends can access particular part of the data, but it is not yet simplified[3].

- With the third party integration it becomes more risky that your information is being shared among various marketers i.e. they may collect your email address from these sites to target you as an audience for their product.[2]

These were few of the privacy issues related to SNS's. By identifying these issues and studying the existing solutions we proposed a privacy framework that will cover most of the points which are not mentioned in the previous research work and yet not implemented in the Social networking sites.

\subsection{Privacy Threats}

According to Bonneau et al. [6], SNS's with enhanced privacy policy have better functionality as well as higher growth rate. They also observed that long time standing SNS's have more extensive privacy methods as compare to newer ones. So it can be concluded that people are more interested in joining SNS's having extensive privacy policy. Threats regarding privacy issues are classified as follows:

- Browsing User Activities- Public personal information on users profile is vulnerable to be downloaded by third parties such as search engines and social network aggregators and simultaneously there is a risk of developing history record of each user over time. Furthermore information about user's online behaviour like visited profiles and also the specification of network settings such as IP address of user's machine may be monitored and logged by Internet Service Provider(ISP) and other similar third parties.

- Disclosing User's Identity- SNS's encourages users to share a profile image. As a result there is a risk that technologies like Content Based Image Retrieval(CBIR) by inspecting the specification of an image could reveal details such as place from which the image was taken. Many people may not be aware of the usage of their personal images or 
videos by other users within SNS's. As in most SNS's users are able to share any images or videos regardless of who is in the images or videos. Therefore there is a high risk of exposing user identity and location even sometimes without their knowledge.

- Cyber-Stalking, Cyber-bullying, harassing, Slandering- The most ideal privacy level is sharing information to only user's friend or group of users. This rule is however vulnerable to cybercriminals who pretend themselves as a friend using fake name and gain access to all information shared by nave users. Many participants desire to be connected to more users and groups which make them insecure to cyber stalking, cyber bullying, harassing.

- Eavesdropping- Millions of users everyday connect to SNS's from mobile devices. It is also expected that many users connect to the Internet from public places through vulnerable wireless access points. Most current SNS's provides insecure communication layer and as a result of these vulnerabilities there is a risk of capturing the transmitted data by sniffing tools.

\subsection{Requirements in Privacy}

Crutz defines privacy as a due that enables users to have 1 . Control of disclosures 2. Control of personal data 3. The right of being left alone. Additionally Cutillo et al[12] state that an ideal SNS's should fulfill the following privacy requirements[12]:

- End-To-End Confidentiality- All interactions are needed to be confidential and only sender and receiver should have access to the data.

- Privacy- Personal information of a user should not be disclosed to any party apart from these explicitly mentioned by the user.

- Access Control- User should be able to manage access control of their profiles as well as attributes of their profiles.

- Authentication- For satisfying the previous requirement receiver of a message should be able to authenticate the sender of the message.

- Data Integrity- For each swapped message whether it is a response or a request, original authentication and also modification detection are needed to be performed.

- Availability- Public data has to be always accessible and all messages should be delivered at any time.

\section{PROPOSED TECHNIQUE}

To best of our knowledge, the current researches do not solve all the privacy risks in SNS's. The focus is on interaction and sharing information between users and concern about users privacy. Solution provide the complete privacy framework that is not applicable to the existing SNS's but are applicable when someone builds a new Social Networking Site, then we can use those privacy policies. Based on the above discussion of threats in SNS's our research is on three things:

1. Exploring Facebook existing privacy policies.

2. Finding vulnerability in existing privacy policy.

3. Proposed enhanced privacy policies that will compete with the existing ones as best as possible.
The enhanced privacy that policies we have proposed and implemented as discussed in the next subsection:

\subsection{Proposed Privacy Policies}

Here we propose certain privacy policies that serve as a resolution for the privacy issues identified in previous section

\section{Policy number 1.}

If a person tags a user on his/her image then user has two options viz. like or unlike. As per new policy, if a user likes the tag then it will appear on user's profile and if he unlike the tags then the tag will completely removed from SNS's like Facebook i.e no other user will be able to see it.

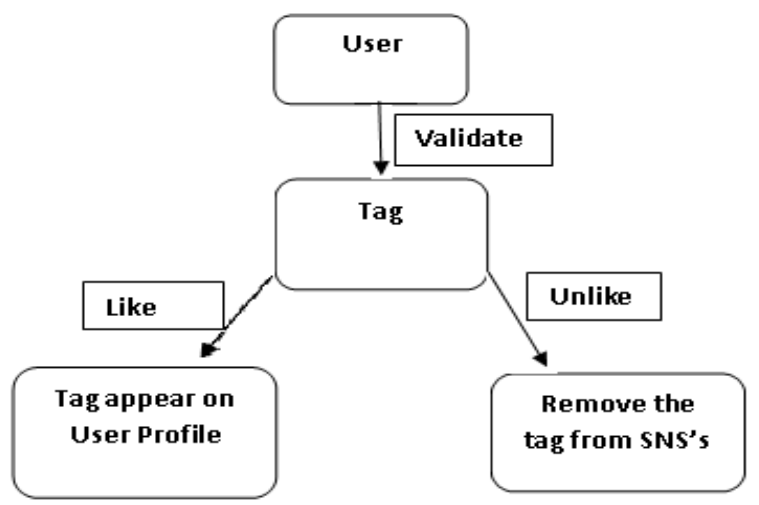

Fig.4.1 Image Tagging Policy

Reason:- User can control the information or access to his data but cannot control what other reveal about him.

Policy number 2.

Disable right click on the image

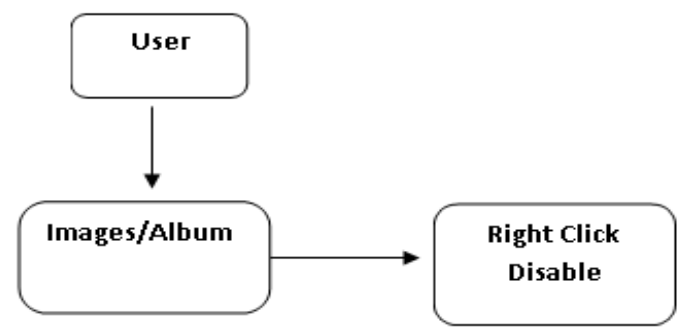

Fig 4.2 Right Click Disable policy

Reason:- The reason for above point is that today we have many tools for editing images. If a user uploads an image and an intruder or friends downloads the image and edit it into something inappropriate then they will harm the reputation of particular user.

Policy number 3.

Provide the user with an option to customize on "Who can post on a user's wall and from whom he/she want to get updates". 


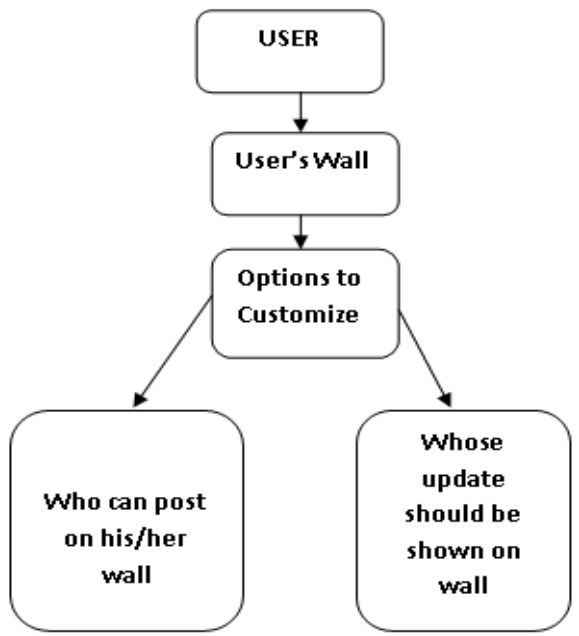

Fig 4.3 Wall Post and Updates Policy

Reason:- Today's SNS's like Facebook provides many options to customize Privacy settings however within that it does not provide the facility to customize Who can post on a user's wall and from whom he/she want to get the updates. There may be people who can post inappropriate content on a user's profile, which needs to be control.

\section{Policy number 4.}

If there is an application which needs our E-mail address to be used, then we should get a warning/notification before proceedings forward so that if we want to proceed we can say Yes otherwise No.

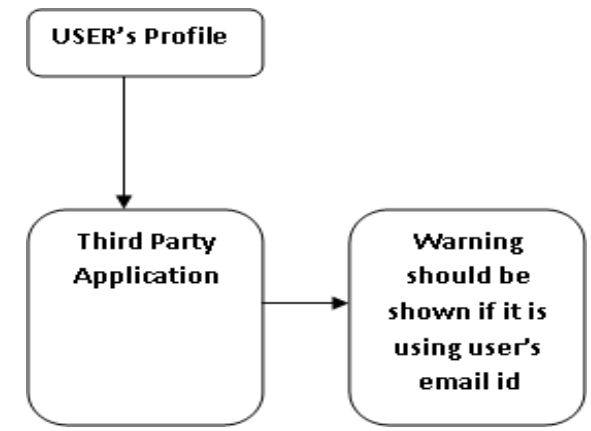

Fig 4.4 Third Party Application Policy

Reason:- Having the third party integration it becomes difficult to identify that any App is using which part of our data and we blindly allow access to these kinds of Apps. As it's after effect our private information may get disclosed. Email address is considered as one of the critical data which many business require to target a specific audience and to increase their productivity.

\section{Policy Number 5.}

Wall Post screening/ filtering for contents being posted by friends is currently not an option for any Facebook user however it becomes a critical and needful requirement in certain scenarios

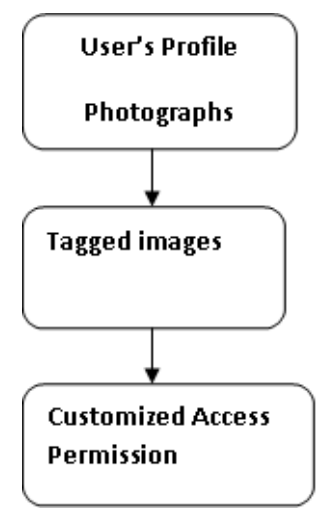

Fig 4.5 Access Customization on Tagged Images Policy

Reason:- By accepting a person as friend we allow them to post contents on our wall. However there may be the casual friends of a user in his/her profile who may not be trusted. There may be occasions when they may post something which is abusive or obscene, inappropriate. To overcome from this problem we propose to use a pool of certain keywords that should be used to screen any content posted by a friend on user's wall.

\section{Policy number 6.}

Option to customize the visibility of comments on the post.

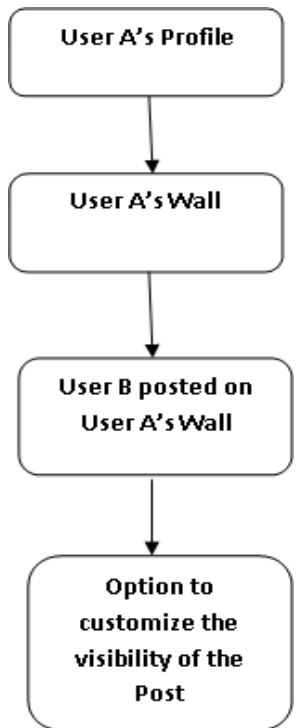

Fig 4.6 Visibility of Comment Policy

Reason:- The friends who are not friends with each other should not be able to view each other's comments on a post updated by their common friend.

\section{Policy number 7.}

Option to customize the visibility of post on other users wall. 


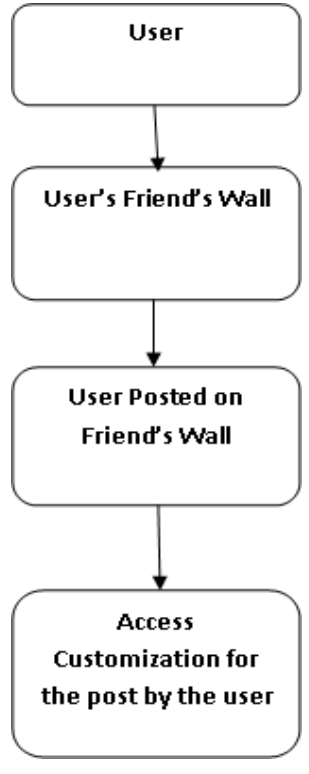

Fig 4.7 Visibility of Post policy

Reason:- When we post any message on a user's wall then the visibility of that post is governed as per the privacy policies of the user on whose wall we posted. However in some situations user who is posting may want to control who among our common friends can view that post. This policy enables us to allow/disallow users among our common friends to view our post.

\section{IMPLEMENTATION}

In this section the privacy policies (proposed in section 4) have been implemented in a Social Networking Sites like Facebook prototype which is developed in php technology. We run this prototype in a UNIX environment having the Xamp Apache Server. This prototype website is not completely mirror image of Facebook but we have tried to make it more similar like Facebook The prototype website is categorized into three major module on which user can interact

$$
\begin{array}{ll}
\text { 1. } & \text { User Module } \\
\text { 2. } & \text { Front Module } \\
\text { 3. } & \text { Back Module }
\end{array}
$$

User Module:- The main purpose of designing this module is to handle all the user related detail.

$$
\begin{array}{ll}
\text { - } & \text { Login Page } \\
\text { - } & \text { Registration Page } \\
\text { Forgot password Page }
\end{array}
$$

Front Module:- The main purpose of this module is to implement all the proposed privacy policies which we have discussed in the earlier sections. The module covers the related detail.

- Chat Module

- Wall Module

Back Module:- The purpose for designing this module is to edit the basic user information like basic information, contact information etc. The module covers the related details
- User Profile

\section{CONCLUSION AND FUTURE WORK}

In this research work, initially examined the existing privacy policies of the most common SNS's like Facebook and tested its policies keeping in mind the queries and requirement of a regular and frequent Facebook user. In this ingoing process their came across few flaws in various privacy policies. Keeping those weakness in mind we have suggested certain modifications in the existing policies. These policies when integrated with the existing policies will help to enhance the privacy policies of Facebook. In support of suggested privacy policies and to test their implementation feasibility we have tried and implemented three of suggested policies in a Facebook like Social Networking site prototype. In Future we tend to intend our privacy policies that offer an easy and communicate with each other and the third party application without revealing much about them. An extended survey is also necessary to validate the efficiency of our privacy framework to protect user privacy.

\section{REFERENCES}

[1] MARK Hachman (Aoril 23,2012). "Facebook Now Totals 1.20 billion Users, Profits Slip". PCMag.com. Retrieved September 24, 2013.

[2] A. Ho, A4. Maiga, and E. Aimeur, "Privacy protection issues in social networking sites,"IEEE/Acs International Conference on Computer Systems and Applications 2009 (AICCSA 2009),PP.271-278,Country,2009

[3] Aimeur, E.; gambus,S.; Ai Ho; , "UPP: User Privacy Policy for Social Networking Sites,"Internet and Web Applications and Services,2009. ICIW '09.Fourth International Conference on vol., no., pp.267-272,24-28 May 2009.

[4] C.Zhang, J.Sun,X.Zhu and Y.Fang," Privacy and security for online social networks: challenges and opportunities," IEEE Network, vol.24,no.4,pp.13-18,2010

[5] Stutzman, F.and Kramer-Duffield, J. Friends only: Examining a Privacy Enhancing behaviour in Facebook. In Proc. CHI'10.ACM Press, 2010.1553-1562.

[6] Thomas,K., Grier, C., and Nicol,D.M.unFriendly: Multi party privacy risks in social networks.In proceedings of the $10^{\text {th }}$ international conference on Privacy enhancing technologies(2010),Soringer-Verlag,pp.236

[7]http://www.facebook.com/privacy

[8] Ai Ho; Maiga, A.; Aimeur, E.; , "Privacy protection issues in social networking sites," Computer Systems and Applications, 2009. AICCSA 2009.IEEE/ACS International Conference on vol.,no., pp.271-278, 10-13 May 2009

[9] Xi Chen; Shuo Shi; ,"A Literature review of Privacy Research on Social Network Sites," Multimedia Information Networking and Security,2009.MINES'09.International Conference on, vol.1,no.,pp.93-97,18-20 Nov.2009

[10] http://www.privacyawarenessweek.org

[11] DotRights Social Networking Page, www.dotrights.org/social-networking 
[12] SeyedHossein Mohtasebi and Ali Dehghantanha," A Mitigation Approach to the Malwares Threats of Social Network Services," Muktimedia Information Networking and Security,2009. MINES'09. International Conference on, vol.1,no.,pp.448-459,2011

[13] Mohammad Mannan, Paul C. Van Oorschot," privacyEnhanced Sharing of Personal Cpntent on the Web," Security And Privacy- Misc" , pp.487-496,April 2125,2008 Beijing, China

[14]Privacy Policy Facebook (2011), www.facebook.com/policy.php

[15]Chi Zhang; Jinyuan Sun;, "Privacy and Security for Online Social networks:Challenges and Opportunities," IEEE Network,Aug.2010

[16] Vorakulpipat, Marks, Rezgui, “ Security and Privacy Issues in Social Networking Sites from User's Viewpoint,'IEEE Network,Jun.2011

[17] P.Kodeswaran, and E.Viegas, "Towards A privacy preserving Policy Based Infrastructure for Social Data
Access To enable Scientific Research,"2010 Eighth Annual International Conference on Privacy,Security and Trust,Jun 2010

[18] I. Polakis and G. Kontaxis," Using Social Networks to harvest Email Addresses," In Proc.CHI"10. ACM Press, 2010

[19] C.Squicciarini and M.Shehab," Privacy policies for shared content in social network sites," In Proc.Chi'10.Acm Press,30 June 2010

[20] Yabing Liu and P. Gummadi, "Analyzing Facebook Privacy Settings:User Expectations vs. Reality," In Proceedings of the $10^{\text {th }}$ international conference on Privacy enhancing technologies(2011)

[21] P.Joshi anf C Kuo," Security and Privacy in Online Social Networks: A Survey",IEEE Network, 2011.

[22] D Michslopoulos and I Mavridis," Surveying Privacy Leaks Through Online Social Networks", $201014^{\text {th }}$ panhellenic Conference on Information, 2010 